\title{
ENERGY EFFICIENT APPROACH BASED ON EVOLUTIONARY ALGORITHM FOR COVERAGE CONTROL IN HETEROGENEOUS WIRELESS SENSOR NETWORKS
}

\author{
Mohamad Nikravan and Seyed Mahdi Jameii \\ Department of Computer Engineering, Shahr-e-Qods Branch, Islamic Azad University, \\ Tehran, Iran
}

\begin{abstract}
ABSTARCT:
Coverage and connectivity are two important requirements in Wireless Sensor Networks (WSNs). In this paper, we address the problem of network coverage and connectivity and propose an energy efficient approach based on genetic evolutionary algorithm for maintaining coverage and connectivity where the sensor nodes can have different sensing ranges and transmission ranges. The proposed algorithm is simulated and it' efficiency is demonstrated via different experiments.
\end{abstract}

\section{KEYWORS}

Wireless Sensor Networks, Coverage, Connectivity, Energy Consumption

\section{INTRODUCTION}

Wireless sensor networks composed of large number of small, low-power wireless sensors. It is believed that WSNs will play a very important role in improving the quality of people's lives [1]. Compared with wired networks, WSNs face several challenges because the sensor nodes have limited resources of energy, processing power and memory [2-3]. In this kind of networks, charging or replacing the battery of the sensors in the network may be difficult or impossible, so energy efficiency is a major problem [4-7]. Due to the resource constraints of the sensors, redundancy of covered area must be reduced for effective utilization of the available resources. An effective approach for energy conservation is sleep scheduling for nodes, while the remaining nodes stay active to provide continuous service and it is not necessary to have all nodes simultaneously operate in the active mode. Keeping only a minimal number of sensors active and putting others into sleep mode is an approach to conserve energy. Sensing coverage is an important issue for WSNs and the goal of it is to have each location in the targeted physical space within sensing range of at least one sensor node. There are many different definitions for sensing coverage. The most popular one defines sensing coverage as the ration of the sensible area to the entire desired area, which means the percentage of the entire area that can be covered by the sensor networks [8]. However, coverage alone in WSNs is not sufficient, and network connectivity should also be considered for the correct operation of WSNs. Connectivity means each sensor node's data is able to be reported to the sink node. Therefore Coverage and Connectivity are other two important requirements in WSNs [9]. 
In this paper, we address the problem of network coverage and connectivity and propose an approach based on non-dominated genetic evolutionary algorithm for maintaining coverage and connectivity where the sensor nodes can have different sensing ranges and transmission ranges, thereby the energy can be saved and network lifetime will be prolonged.

The remaining of this paper is organized as follows. Section 2 introduces some of the previous related works. In section 3, the proposed solution is presented. In section 4 , we provide the simulation results and comparisons are described, and finally section 5 concludes the paper.

\section{RELATED WORKS}

In recent years, a lot of studies have been done for addressing the coverage and connectivity issues in WSNs. Authors of [10] studied the impact of sensor node distribution on the network coverage and mentioned that an important way to deal with coverage, connectivity, and lifetime maximization is to scheduling the sensor nodes after deploying a densely distributed sensor network randomly in area. OGDC (Open Geographic Density Control) [11] is one of the popular coverage algorithms for WSNs. In this approach, the energy is conserved by controlling the density of the active nodes but it considers the deterministic placements of the nodes, which is possible only for the small scale networks. CCP (Coverage Configuration Protocol) which is proposed in [12], network can be configured to maintain varying degrees of coverage. Initially, all the nodes in the network remain in the 'OFF' state. Each node executes eligibility algorithm, to decide if it should go to the 'ON' state, or to stay back in the 'OFF' state. The algorithm considers the coverage degree of all the intersection points of the sensing disks in its sensing range. This algorithm do not require deterministic placement of nodes, but they do not consider the trade-off between the sensor energy and network coverage. In ADS (Area Dominating Set) algorithm [13], the smallest possible subset of nodes is activated to fully cover a monitoring area. This algorithm is based on a Connected Dominating Set (CDS) [14] algorithm. A CDS is a connected subset of a graph such that every vertex in the graph is either in the set or adjacent to a vertex in the set. The CDS protocol is used to maintain the connectivity of the network. In this algorithm, periodic sending of broadcast messages, and listening for the reply messages are required, so increases the number of message exchanges between the nodes and the network lifetime will be decreased. Author of [15] proposed a multi-objective optimization approach for optimizing the energy consumption and coverage in WSNs. In [16], the sensing ranges of sensor nodes are adjustable and the impact of this adjustment on coverage is studied. In this algorithm, for prolonging the network lifetime, each node prefers to operate in minimum sensing range considering the sensing coverage. The research presented in [17] used the characteristics of voronoi diagram and direction-adjustable directional sensors and proposed a distributed greedy algorithm which can improve the effective field coverage of directional sensor networks. Multi ebjective optimization algorithm presented in [18] efficiently schedules the sensor nodes of a WSN and optimizes energy consumption, lifetime, and coverage. Authors of [19] formulated the sensor node deployment task as a multi-objective optimization problem. The aim of this optimization approach is to find a deployed sensor node arrangement for maximizing the area of coverage and minimize the number of deployed sensor nodes. 
International Journal of Computer Science, Engineering and Information Technology (IJCSEIT), Vol. 4, No.2, April 2014

\section{PROPOSED PROTOCOL}

In this section, we present a detailed description of the proposed multi-objective optimization approach. The proposed approach is based genetic evolutionary algorithm and will be executed by the sink node. The final results obtained by the sink node are sent to sensor nodes and each sensor nodes will adjust its transmission range, sensing range and scheduling state. Our WSN composed of $\mathrm{N}$ stationary resource constraint sensor nodes and a static resource-rich sink. The sensor nodes deployed randomly with uniform distribution over a finite, two-dimensional region. Each node can adjust its transmission range and sensing range. We assume that each node knows its position. For modeling the coverage in WSN, we assume the monitoring area is divided into a set of points. The coverage area of each sensor node is modeled as a circle of radius $R_{s}$. If the Euclidean distance between a point $P$ and a sensor node $S_{j}$ is lower than $R_{s}$, then $P$ can be covered by $\mathrm{S}_{\mathrm{j}}$. For any point $\mathrm{P}$ at $(\mathrm{x}, \mathrm{y})$, we denote the Euclidean distance between $\mathrm{Si}$ and $\mathrm{P}$ as follow:

$$
\mathrm{d}\left(\mathrm{S}_{\mathrm{i}}, \mathrm{P}\right)=\left(\mathrm{x}_{\mathrm{i}}-\mathrm{x}\right)^{2}+\left(\mathrm{y}_{\mathrm{i}}-\mathrm{y}\right)
$$

The Equation (2) expresses the coverage state of point $\mathrm{P}_{\mathrm{i}}$ by sensor $\mathrm{Si}$ :

$$
\operatorname{COV}_{x y}\left(S_{i}\right)=\left\{\begin{array}{rr}
1 & \text { if } d\left(S_{i}, P_{i}\right)<R_{s} \\
0 & \text { Otherwise, }
\end{array}\right.
$$

The coverage in the case that a region is overlapped by a set of $\mathrm{N}_{\text {active }}$ sensor nodes $\left(\mathrm{N}_{\text {active }} \subseteq N\right)$ is given by Equation (3):

$$
\operatorname{COV}_{x y}\left(N_{\text {active }}\right)=\cup_{S_{i} \in N_{\text {active }}} \operatorname{COV}_{x y}\left(S_{i}\right)
$$

The decision variables, objectives and constrains of this multi-objective problem are as follow:

Decision variables:

$R_{S}(i)$ : the sensing range of the sensor node $i$.

$\mathrm{R}_{\mathrm{t}}(\mathrm{i})$ : the transmission range of the sensor node $i$.

State $S_{\mathrm{i}}$ : the scheduling state of the sensor node $\mathrm{i}$.

Objectives:

Objective 1: Maximizing the coverage rate of the entire region by sensor set $\mathrm{N}_{\text {active }}$ which is defined as follow:

$$
\text { Coverage Rate }=\frac{\left[\sum_{x=1}^{m} \sum_{k=1}^{n} \operatorname{cov}_{x, y}\left(N_{\text {active }}\right)\right]}{(m \times n)}
$$

Objective 2 : Minimizing the number of active nodes $\left(\mathrm{N}_{\text {active }}\right)$

For converting this objective to a maximization objective, we consider the number of inactivated sensors which is calculated as follow:

Objective 3: Maximizing the network lifetime.

$$
\mathrm{N}_{\text {inactive }}=\mathrm{N}-\mathrm{N}_{\text {active }}
$$


We consider the residual energy of the sensor nodes and maximize the sum of the residual energy of nodes for maximizing the network lifetime:

Constrain: The network connectivity.

The network is said to be connected if any node can communicate with any other node.

So, the optimization problem is defined as follow:

$$
\mathrm{Z}=\operatorname{argmax}(\text { objective } 1 \text {, objective } 2 \text {, objective } 3 \text { ) }
$$

where the connectivity constrain should be satisfied.

Each solution consists of $\mathrm{N}$ items. Each item represents an individual sensor node $\mathrm{j}$ and consists of the scheduling status, transmission range and sensing range of the sensor node $j$ and represent as Status $(j), R_{s}(j)$ and $R_{t}(j)$ respectively. So, we need five bit for each item: the first bit is used to describe the scheduling status of the sensor node while the 0 value means the corresponding sensor node is inactive and 1 value means it is active. The four remaining bits demonstrate the sensing range and transmission range of the sensor node ( 2 bit for transmission range and 2 bit for sensing range). So, each sensor node may operate in one of the four $\left(2^{2}\right)$ predefined transmission range level and four $\left(2^{2}\right)$ sensing range level. This genetic representation of solution is depicted in figure 1.

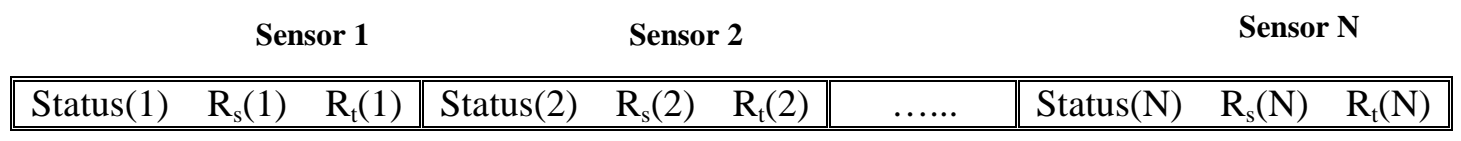

Figure 1. Genetic representation of the solution

The proposed approach to solve multi-objective problem is described in the following steps:

Step 1: Choose population size $\mathrm{K}$, crossover and mutation probability, crossover and mutation index and maximum number of generations.

Step 2: Generate a random initial population $\mathrm{Pop}_{0}$. Set the generation count $\mathrm{t}=0$.

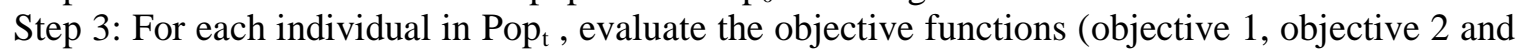
objective 3) and constraint violations.

Step 4: Create offspring population Off $_{t+1}$ from Pop ${ }_{t+1}$ by using the crowded tournament selection, crossover and mutation operators as presented in [20].

Step 5: Combine the Off $\mathrm{t}_{\mathrm{t}+1}$ and Pop $\mathrm{t}_{\mathrm{t}+1}$ to create combine population $\mathrm{R}_{\mathrm{t}}$.

Step 6: Perform non-dominated sorting over $\mathrm{R}_{\mathrm{t}}$ and identify different fronts $\mathrm{PFi}=1 ; 2 ; \ldots$; etc.

Step 7: If the size of non-dominated set $\mathrm{M}$ is greater than the population size $\mathrm{N}$, then remove the $\mathrm{M} \mathrm{N}$ individuals from non-dominated set by using DCD based strategy as [21], elsewhere, go to step 4.

Step 8: If the algorithm reaches the maximum generation count, then stop the algorithm, otherwise, increment generation count $(t=t+1)$ and go to step 3 . The non-dominated individuals in $\mathrm{Pop}_{\mathrm{t}}$ are the Pareto-optimal front and the solutions of the problem. 


\section{SIMULATION RESULTS}

The proposed algorithm is simulated via NS-2 simulator and different experiments with different number of deployed sensors are conducted. The results of this simulation are depicted through different diagrams and the proposed algorithm is compared with OGDC [11] and RAA-2L [16] algorithms. We used the IEEE 802.11 as Mac protocol and the initial energy of the sensors are 2 J. All simulations are repeated 30 times and the average of the results are depicted. The size of the region is $100 * 100 \mathrm{~m}^{2}$. The predefined values of sensing and the transmission ranges are $(5,10,15,20)$ and $(10,20,30,40)$ respectively. The genetic parameters are considered as follow:

Maximum number of generations $=200$

Population size $=100$

Crossover probability $=0.9$

Mutation probability $=0.1$

In the first experiment, the average of transition radiuses of nodes at the different generations of the proposed algorithm is measured. As can be seen in figure 2, the average of the transmission radiuses is less than the maximum transmission radius (less than 40). Also, the average of transmission radiuses of nodes at generation 200 is less rather than generation 100. This is because the proposed algorithm at the generation 200 reaches to stable state.

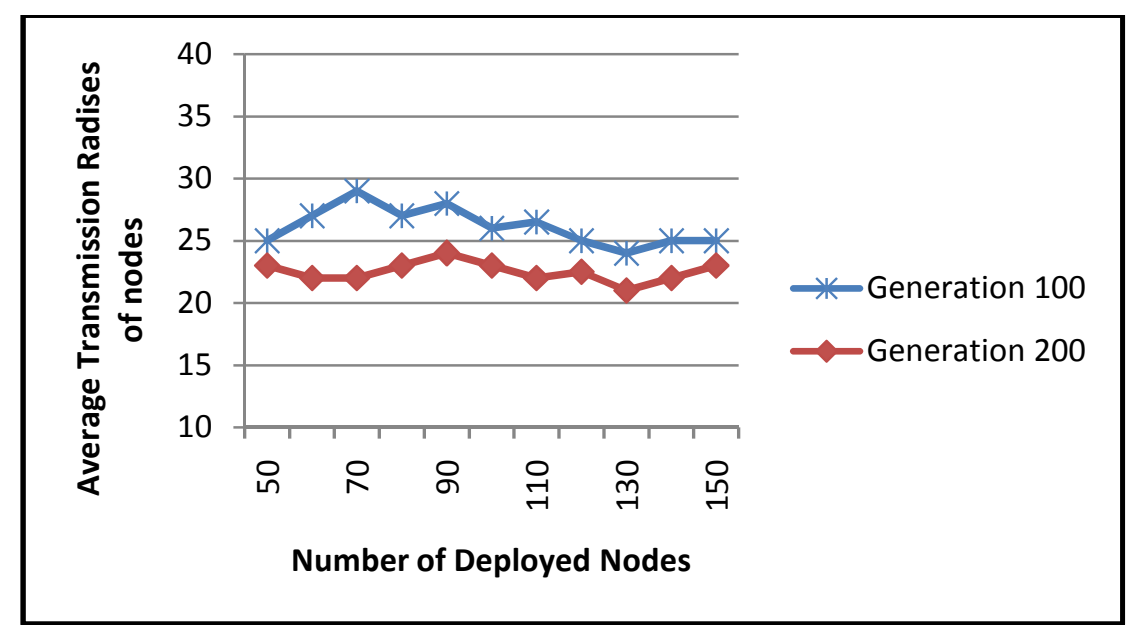

Figure 2: Average transmission radiuses of nodes

In the second experiment, different network size is considered and the coverage rate is evaluated according to Equation 4. The results of this experiment are depicted in figure 3 and the proposed algorithm is compared with OGDC and RAA-2L algorithms. As can be seen, we observe that with the same number of active sensors, the proposed algorithm obtains higher coverage rate than other algorithms. In the case of low density of active sensors, OGDC provides the minimum coverage rate as compared to the other protocols. This is due to the fact that OGDC suffers from the many redundant active sensors. 


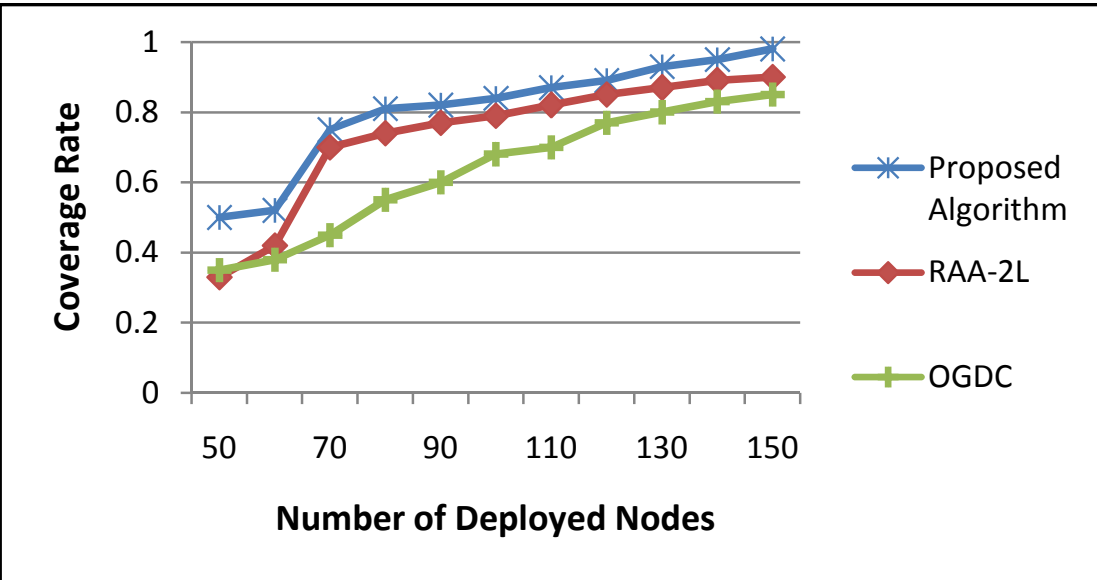

Figure 3: Coverage rate Vs. number of nodes

In the third experiment, total remaining energy of nodes in different configurations on network is measured for the proposed algorithm and is compared with the OGDC and RAA-2L algorithms. As can be seen in figure 4, total remaining energy of nodes in the proposed algorithm is higher than other algorithms. This is because the proposed algorithm can adjust the transmission range and the sensing ranges of nodes and so, the total energy consumption will be decreased.

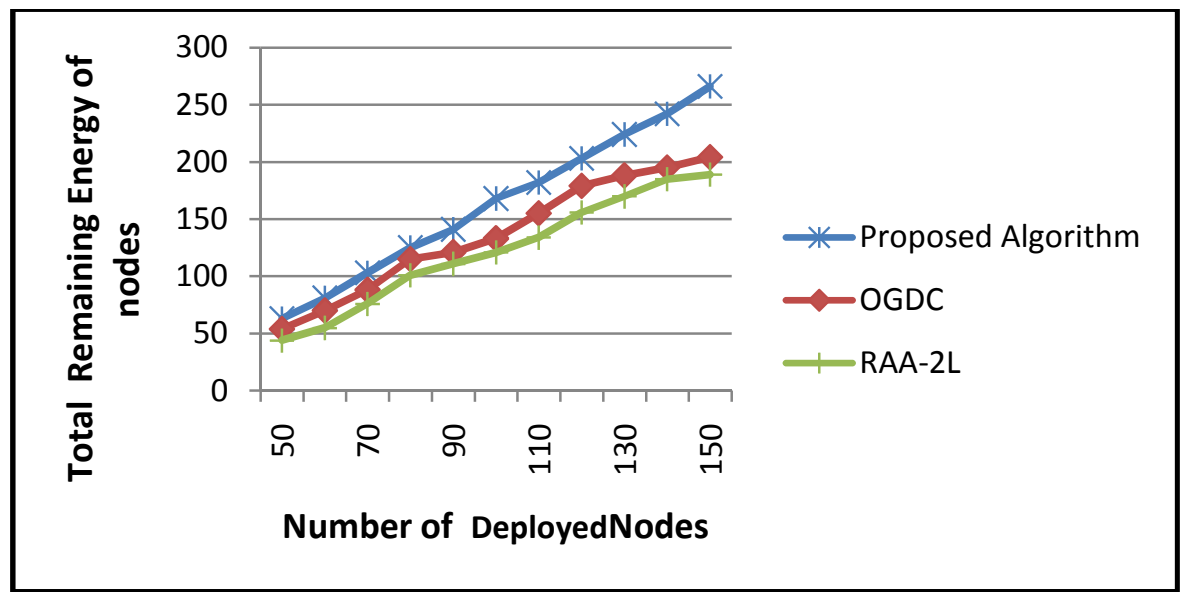

Figure 4: Total remaining energy of nodes Vs. number of deployed nodes

\section{Conclusions}

In this paper, we proposed an energy efficient approach based on genetic evolutionary algorithm for addressing the problem of coverage and connectivity issues in heterogeneous wireless sensor network where the sensor nodes had different sensing ranges and transmission ranges. We considered different metrics in the proposed optimization approach. The proposed algorithm was simulated and compared with the OGDC and RAA-2L algorithms. The obtained results demonstrated the efficiency of the proposed algorithm in terms of average transition radiuses of nodes, coverage rate and total remaining energy of nodes. 
International Journal of Computer Science, Engineering and Information Technology (IJCSEIT), Vol. 4, No.2, April 2014

\section{ACKNOWLEDGEMENTS}

The authors wish to thank Islamic Azad University, Shahr-e-Qods branch for supporting this work through grants.

\section{REFERENCES}

[1] GY Liu, B. Xu, H. Chen, (2012) "Decentralized estimation over noisy channels in cluster-based wireless sensor networks", International Journal of Communication Systems; Vol. 25, No. 10, pp.1313-1329.

[2] A . Peiravi,H. Mashhadi, SH. Javadi, (2013) "An optimal energy-efficient clustering method in wireless sensor networks using multi-objective genetic algorithm", International Journal of Communication Systems, Vol. 26, No. 1, pp. 114-126.

[3] HJ Huang, GM. Hu, FC. Yu, (2013) "Energy-aware geographic routing in wireless sensor networks with anchor nodes", International Journal of Communication Systems, Vol. 26, No. 1, pp. 100-113.

[4] KW. Jang, (2012) "Meta-heuristic algorithms for channel scheduling problem in wireless sensor networks", International Journal of Communication Systems, Vol. 25, No. 4, pp.427-446.

[5] H. Tan, I. Korpeoglu, and I. Stojmenovic, (2011) "Computing Localized Power Efficient Data Aggregation Trees for Sensor Networks," IEEE Trans. Parallel and Distributed Systems, Vol. 22, No. 3, pp. 489-500.

[6] Y. Wu and Y. Li, (2008) "Construction Algorithms for k-Connected Dominating Sets in Wireless Sensor Networks," Proc. ACM Int'l Symp. Mobile Ad Hoc Networking and Computing (MobiHoc).

[7] Z. Yu, X. Bai, D. Xuan, and W. Jia, (2011) "Connected Coverage in Wireless Networks with Directional Antennas," Proc. IEEE INFOCOM.

[8] Y.R. Tsai, (2008) 'Sensing coverage for randomly distributed wireless sensor networks in shadowed environments', IEEE Trans. Veh. Technol, Vol. 57, No. 1, pp. 556-564.

[9] M . CARDEI, S. YANG, J. WU (2008) 'Algorithms for fault-tolerant topology in heterogeneous wireless sensor networks', IEEE Trans. Parallel Distrib. Syst., Vol. (19), No. (3), pp. 545-558.

[10] M. Peng, H. Chen, Y. Xiao, S. Ozdemir, A. V. Vasilakos, and J. Wu, (2011) "Impacts of sensor node distributions on coverage in sensor networks," J. Parallel Distrib. Comput., vol. 71, no. 12, pp. 15781591.

[11] H. Zhang, J. Hou, (2005) "Maintaining sensing coverage and connectivity in large sensor networks" Wireless Ad Hoc and Sensor Networks: An International Journal, Vol. 1, No.(2), pp. 89-124.

[12] X. Wang, G. Xing, Y. Zhang, C. Lu, R. Pless, C. Gill, (2005) "Integrated coverage and connectivity configuration in wireless sensor networks", ACM Transactions on Sensor Networks Vol. 1, No. 1.

[13] J. Carle, D. Simplot-Ryl, (2004) "Energy efficient area monitoring for sensor networks", Computer, Vol. 37, No. 2, pp. 40-46.

[14] P. Wan, K.M. Alzoubi, O. Frieder, (2004) "Distributed construction of connected dominating set in wireless ad hoc networks, Mobile Networks and Applications", Vol. 9, No. 2, pp. 141-149.

[15] H.Z . Abidin, N.M. Din, Y.E. Jalil, (2013) "Multi-objective Optimization (MOO) Approach for Sensor Node Placement in WSN", IEEE 7th International Conference on Signal Processing and Communication Systems (ICSPCS), Carrara.

[16] A. Venuturumilli, (2006) "Obtaining Robust Wireless Sensor Networks Throuh Self-Organization of Heterogeneous Connectivity", In: Proceedings of the International Conference on Complex Systems (ICCS'06), Boston, MA.

[17] T.W. Sungn, C. S. Yang, (2014), "Voronoi-based coverage improvement approach for wireless directional sensor networks", Journal of Network and Computer Applications 2014;Vol. 39, No. 1, pp. 202-213.

[18] S. Sengupta, S. Das, M. Nasir, A. V. Vasilakos, and W. Pedrycz, (2012) "An Evolutionary Multiobjective Sleep-Scheduling Scheme for Differentiated Coverage in Wireless Sensor Networks", IEEE Transactions on Systems, Man, and Sybernetics- Part C: Applications and Reviews; Vol. 42, No. 6. 
International Journal of Computer Science, Engineering and Information Technology (IJCSEIT), Vol. 4, No.2, April 2014

[19] S. Sengupta , S. Das, M. Nasir, B. K. Panigrahi, (2013) "Multi-objective node deployment in WSNs: In search of an optimal trade-off among coverage, lifetime, energy consumption, and connectivity", Engineering Applications of Artificial Intelligence, Vo. 26, No. 1, pp. 405-416.

[20] S. Kannan, S. Baskar, J.D. Mccalley, P. Murugan, (2009) "Application of NSGA-II algorithm to generation expansion planning”, IEEE Trans Power Syst, Vol. 24, No. 1, pp.454-61.

[21] B. Luo, J. Zheng, j. Xie, J. Wu, (2008) ; Dynamic crowding distance - a new diversity maintenance strategy for MOEAs", In: Proceedings of the IEEE international conference on natural computation, China. 